\title{
AN EXISTENCE THEOREM IN POTENTIAL THEORY
}

\author{
MASANORI KISHI
}

Dedicated to the memory of Professor TaDasi Nakayama

1. Concerning a positive lower semicontinuous kernel $G$ on a locally compact Hausdorff space $X$ the following existence theorem was obtained in [3].

TheOREM A. Assume that the adjoint kernel $\breve{G}$ satisfies the continuity principle. Then for any separable compact subset $K$ of $X$ and any positive upper semicontinuous function $u(x)$ on $K$, there exists a positive measure $\mu$, supported by K, such that

$$
\begin{array}{ll}
G \mu(x) \geq u(x) & \text { G.p.p.p. on } K, \\
G \mu(x) \leq u(x) & \text { on } S \mu, \text { the support of } \mu .
\end{array}
$$

Nakai [4] proved the theorem without assuming the separablility of $K$. Using Kakutani's fixed-point theorem he simplified a part of the proof. But he needed prudent considerations on topology in order to avoid the separability. In this paper we shall give a simpler proof of the theorem without assuming the separability. We shall deal with a slightly more general kernel and use Glicksberg-Fan's fixed-point theorem.

2. A lower semicontinuous function $G(x, y)$ on $X \times X$ with $0 \leq G(x, y) \leq+\infty$ is called a non-negative 1.s.c. kernel on $X$. The kernel $G$, defined by $\check{G}(x, y)$ $=G(y, x)$, is called the adjoint kernel of $G$. The potential $G \mu(x)$ of a positive measure $\mu$ is defined by $G \mu(x)=\int G(x, y) d \mu(y)$. The adjoint potential $\breve{G} \mu(x)$ is similarly defined. The adjoint kernel $\breve{G}$ is said to satisfy the continiuity principle when finite continuous is every adjoint potential $\breve{G} \mu$ of a positive measure $\mu$ with compact support which is finite continuous as a function on $S \mu$.

3. We shall prove

Received April 12, 1965. 
Theorem B. Let $G$ be a non-negative l.s.c. kernel on a locally compact Hausdorff space $X$. Assume that $G(x, x)>0$ for any $x \in X$ and the adjoint kernel $G$ satisfies the continuity principle. Then for any compact subset $K$ of $X$ and any positive finite upper semicontinuous function $u(x)$ on $K$, there exists a positive measure $\mu$, supported by $K$, such that

$$
\begin{array}{ll}
G \mu \geq u & G-p . p . p . \text { on } K^{1)}, \\
G \mu \leq u & \text { on } S \mu .
\end{array}
$$

4. First we prove

Theorem $C$. If $G$ is a non-negative finite continuous kernel on a compact Hausdorff space $K$ such that $G(x, x)>0$ on $K$, there exists a positive measure $\mu$ on $K$ such that

$$
\begin{aligned}
& G \mu(x) \geq 1 \text { on } K, \\
& G \mu(x)=1 \text { oin } S \mu .
\end{aligned}
$$

Proof. Denote by $\mathscr{M}_{1}(K)$ the totality of positive unit measures on $K$. This, with the vague topology, is compact and convex. We define a point-toset mapping $\varphi$ on $\mathscr{M}_{1}(K)$ as follows: we put, for any $\mu \in \mathscr{M}_{1}(K)$,

$$
\varphi(\mu)=\left\{\nu \in \mathscr{M}_{1}(K) ; \int G \mu d \nu=\inf _{\lambda \in \mathscr{M}_{1}(K)} \int G \mu d \lambda\right\}
$$

Since $G(x, y)$ is finite continuous, $\varphi(\mu)$ is non-empty and convex, and the mapping $\varphi: \mu \rightarrow \varphi(\mu)$ is closed in the following sense : if nets $\left\{\mu_{\alpha} ; \alpha \in D\right.$, a directed set $\}$ and $\left\{\nu_{\alpha} ; \alpha \in D\right\}$ converge vaguely to $\mu$ and $\nu$ respectively and if $\nu_{\alpha} \in \varphi\left(\mu_{\alpha}\right)$ for any $\alpha \in D$, then $\nu \in \varphi(\mu)$. Consequently by Glicksberg-Fan's fixed-point theorem $^{21}$ there exists a measure $\mu_{0} \in \mathscr{M}_{1}(K)$ such that $\mu_{0} \in \varphi\left(\mu_{0}\right)$. Then $m_{0}=\int G \mu_{0} d \mu_{0}=\inf _{\lambda \in{ }_{1}(K)} \int G \mu_{0} d \lambda$ does not vanish, since $G(x, x)>0$ on $K$. The measure $\mu=m_{0}^{-1} \mu_{0}$ fulfills all the requirements.

5. Using Theorem $\mathrm{C}$ we prove

TheOREM D. If $G$ is a non-negative l.s.c. kernel on a compact Hausdorff space $K$ such that $G(x, x)>0$ for any $x \in K$ and if the adjoint kernel $\check{G}$ satisfies

1) This means that every compact subset of the exceptional set $\{x \in K ; G \mu(x)<u(x)\}$ does not support any positive measure $\lambda \neq 0$ such that $\int G \lambda d \lambda<\infty$.

2) Cf. [1] and [2]. 
the continuity principle, then there exists a positive measure $\mu$ on $K$ such that

$$
\begin{array}{ll}
G \mu \geq 1 & \text { G-p.p.p. on } K, \\
G \mu \leq 1 & \text { on } S \mu .
\end{array}
$$

Proof. Put $m=\inf _{x \in K} G(x, x)>0$, and take a finite number of open neighborhoods $U_{i}(1 \leq i \leq N)$ such that $\bigcup_{1}^{N} U_{i} \supset K$ and $G(x, y)>\frac{1}{2} m$ in $U_{i} \times U_{i}$. There exists an increasing net $\left\{G_{\alpha} ; \alpha \in D\right.$, a directed set $\}$ of non-negative finite continuous functions $G_{\alpha}(x, y)$ on $K \times K$ such that $G_{\alpha}(x, y)>\frac{1}{2} m$ in $\bigcup_{1}^{N} U_{i} \times U_{i}$ and $\lim _{D} G_{\alpha}(x, y)=G(x, y)$ at any point $(x, y) \in K \times K$. Then by Theorem B there exists a positive measure $\mu_{\alpha}$ on $K$ such that $G_{\alpha} \mu_{\alpha} \geq 1$ on $K$ and $G_{\alpha} \mu_{\alpha}=1$ on $S \mu_{\alpha}$. The net $\left\{\mu_{\alpha} ; \alpha \in D\right\}$ is bounded. In fact, for a point $x \in S \mu_{\alpha} \cap U_{i}$,

$$
\begin{aligned}
1 & =G_{\alpha} \mu_{\alpha}(x)=\int G_{\alpha}(x, y) d \mu_{\alpha}(y) \\
\geq & \int_{U_{i}} G_{\alpha}(x, y) d \mu_{\alpha}(y)>\frac{1}{2} m \mu_{\alpha}\left(U_{i}\right),
\end{aligned}
$$

and hence $\mu_{\alpha}\left(U_{i}\right) \leq \frac{2}{m}$ and $\mu_{\alpha}(K) \leq \frac{2 N}{m}$. Thus there exists a cluster point $\mu$. Put

$$
D^{\prime}=\left\{\alpha^{\prime}=\langle\alpha, \omega\rangle ; \omega \text {, a vague neighborhood of } \mu \text { containing } \mu_{\alpha}\right\} .
$$

Then $D^{\prime}$ is a directed set with the natural order. Putting, for $\alpha^{\prime}=\langle\alpha, \omega\rangle \in D^{\prime}$, $\mu_{\alpha^{\prime}}=\mu_{\alpha}$ and $G_{\alpha^{\prime}}=G_{\alpha}$, we see that $\mu_{\alpha^{\prime}} \rightarrow \mu$ vaguely and $G_{\alpha^{\prime}}(x, y) \nearrow G(x, y)$ at any point $(x, y) \in K \times K$. We shall show the validity of (i) and (ii) for $\mu$.

Proof of (i). Suppose that there exists a positive measure $\lambda \neq 0$ such that $S_{\lambda} \subset\{x \in K ; G \mu(x)<1\}$ and $\int \breve{G} \lambda d \lambda<\infty$. Since $\breve{G}$ satisfies the continuity principle, we may assume that $\check{G} \lambda$ is finite continuous on $K$. Hence

$$
\begin{aligned}
\int d \lambda>\int G \mu d \lambda & =\int \check{G} \lambda d \mu=\lim _{D^{\prime}} \int \check{G} \lambda d \mu_{\alpha^{\prime}} \\
& =\lim _{D^{\prime}} \int G \mu_{\alpha^{\prime}} d \lambda \geq \lim \sup _{D^{\prime}} \int G_{\alpha^{\prime}} \mu_{\alpha^{\prime}} d \lambda \geq \int d \lambda .
\end{aligned}
$$

Proof of (ii). Let $x_{0}$ be an arbitrary fixed point on $S_{\mu}$, and put $D^{\prime \prime}=\left\{\alpha^{\prime \prime}=\left\langle\alpha^{\prime}, U\right\rangle ; U\right.$, a neighborhood of $x_{0}$ containing a point $x_{\alpha^{\prime}}$ of $\left.S \mu_{\alpha^{\prime}}\right\}$.

This is a directed set with the natural order. Putting, for $\alpha^{\prime \prime}=\left\langle\alpha^{\prime}, U\right\rangle \in D^{\prime \prime}$, $x_{\alpha^{\prime \prime}}=x_{\alpha^{\prime}}, \mu_{\alpha^{\prime \prime}}=\mu_{\alpha^{\prime}}$ and $G_{\alpha^{\prime \prime}}=G_{\alpha^{\prime}}$, we see that $x_{\alpha^{\prime \prime}} \rightarrow x_{0}, \mu_{\alpha^{\prime \prime}} \rightarrow \mu_{0}$ and $G_{\alpha^{\prime \prime}}(x, y) \nearrow$ 
$G(x, y)$ along $D^{\prime \prime}$. Hence for any $\alpha_{0}^{\prime \prime} \in D^{\prime \prime}$

$$
1=\lim _{D^{\prime \prime}} G_{\alpha^{\prime}} \mu_{\alpha^{\prime \prime}}\left(x_{\alpha^{\prime \prime}}\right) \geq \lim _{D^{\prime \prime}} G_{\alpha_{0}^{\prime \prime}} \mu_{\alpha^{\prime \prime}}\left(x_{\alpha^{\prime \prime}}\right)=G_{\alpha_{0}^{\prime \prime}} \mu\left(x_{0}\right) .
$$

Consequently $G \mu\left(x_{0}\right)=\lim _{D^{\prime \prime}} G_{\alpha^{\prime}} \mu\left(x_{0}\right) \leq 1$.

6. From Theorem $D$ follows immediately

Theorem E. Let $G$ be a non-negative l.s.c. kernel on $X$ such that $G(x, x)$ $>0$ for any $x \in X$ and the adjoint kernel $\breve{G}$ satisfies the continuity principle. Then for any positive finite continuous function $u(x)$ on a compact set $K$, there exists a positive measure $\mu$, supported by $K$, such that

$$
\begin{array}{ll}
G \mu(x) \geq u(x) & \text { G-p.p.p. on } K, \\
G \mu(x) \leq u(x) & \text { on } S_{\mu} .
\end{array}
$$

In fact, $G^{\prime}(x, y)=G(x, y) / u(x)$ is a non-negative 1.s.c. kernel on $K$, the adjoint kernel of which satisfies the continuity principle. Hence by Theorem D there exists a positive measure $\mu$ on $K$ such that

$$
\begin{array}{ll}
G^{\prime} \mu \geq 1 & G^{\prime}-p . p . p . \text { on } K, \\
G^{\prime} \mu \leq 1 & \text { on } S_{\mu} .
\end{array}
$$

This $\mu$ fulfills the requirements of Theorem E.

7. Now we can prove Theorem B. Let $\left\{\boldsymbol{u}_{\alpha}(x) ; \alpha \in D\right\}$ be a decreasing net of positive finite continuous functions on $K$ such that $u_{\alpha}(x) \searrow u(x)$. Then there exists a positive measure $\mu_{\alpha}$ on $K$ such that

$$
\begin{array}{ll}
G \mu_{\alpha}(x) \geq u_{\alpha}(x) & G-p . p . p . \text { on } K \\
G \mu_{\alpha}(x) \leq u_{\alpha}(x) & \text { on } S \mu_{\alpha} .
\end{array}
$$

The net $\left\{\mu_{\alpha}\right\}$ is bounded, and similarly as in the proof of Theorem $\mathrm{D}$, a subnet converges vaguely to a cluster point $\mu$ of the net $\left\{\mu_{\alpha}\right\}$. This $\mu$ fulfills the requirements of Theorem $B$.

\section{REFERENCES}

[1] K. Fan, Fixed-point and minimax theorems in locally convex topological linear spaces, Proc. Nat. Acad. Sci. U.S.A., 38 (1952), 121-126.

[2] I. L. Glicksberg, A further generalization of the Kakutani fixed point theorem, with application to Nash equilibrium points, Proc. Amer. Math. Soc., 3 (1952), 170-174. 
[ 3 ] M. Kishi. Maximum principles in the potential theory, Nagoya Math. J., 23 (1963), 165-187.

[ 4] M. Nakai, On the fundamental existence theorem of Kishi, Nagoya Math. J., 23 (1963), "189-198.

Mathematical Institute

Nagoya University 